\title{
A Case of Refractory Generalized Myasthenia Gravis Treated with Polypharmacy and Plasma Exchange
}

\author{
Koushun Matsuo ${ }^{1}$, Chihiro Fujii ${ }^{1,3}$, Kiyonori Miyata $^{1}$, Yuka Kawasaki Segawa ${ }^{2}$, Hiroshi Kado ${ }^{2,4}$, Tsuguru Hatta ${ }^{2}$, Yu Sato $^{3}$, Rei Yasuda $^{3}$, Masanori Nakagawa ${ }^{3,5}$ \\ Toshiki Mizuno ${ }^{3}$ \\ ${ }^{1}$ Division of Neurology, Ohmihachiman Community Medical Center, Ohmihachiman, Shiga, Japan \\ ${ }^{2}$ Division of Nephrology, Ohmihachiman Community Medical Center, Ohmihachiman, Shiga, Japan \\ ${ }^{3}$ Department of Neurology, Graduate School of Medical Science, Kyoto Prefectural University of Medicine, Kyoto, Japan \\ ${ }^{4}$ Department of Nephrology, Graduate School of Medical Science, Kyoto Prefectural University of Medicine, Kyoto, Japan \\ ${ }^{5}$ Department of Neurology, North Medical Center, Kyoto Prefectural University of Medicine, Kyoto, Japan \\ "Corresponding author: Koushun Matsuo, Division of Neurology, Ohmihachiman Community Medical Center, Tsuchidacho 1379, Ohmihachiman, Shiga 523-0082, \\ Japan, Tel: +81-748-33-3151; Fax+81-748-33-4877; E-mail: matsuo@pb4.so-net.ne.jp \\ Received date: January 13, 2016; Accepted date: April 12, 2016; Published date: April 19, 2016 \\ Copyright: ( 2016 Matsuo K, et al. This is an open-access article distributed under the terms of the Creative Commons Attribution License, which permits unrestricted \\ use, distribution, and reproduction in any medium, provided the original author and source are credited.

\begin{abstract}
A 62-year-old man with myasthenia gravis (MG) was admitted with dyspnea, bulbar symptoms (dysphagia and dysarthria), and subsequent muscular weakness of the limbs. Results for anti-acetylcholine receptor antibody and muscle-specific kinase antibody were negative. We introduced periodic plasma exchange as a new therapeutic strategy. Once double-filtration plasmapheresis (DFPP) was adopted, effects became notable, but the response shortened over time. After stepwise increases in PSL up to $60 \mathrm{mg} /$ day with insulin, the interval between DFPP was prolonged from 1 week to 2 weeks. DFPP was significantly beneficial in this case. Combining several effective methods can optimize therapeutic effects against refractory MG.
\end{abstract}

Keywords: Refractory generalized myasthenia gravis; Doublefiltration plasmapheresis (DFPP); Polypharmacy; Double seronegative type

\section{Introduction}

Myasthenia gravis (MG) is characterized by a clinical presentation of muscle fatigability and weakness. These symptoms of MG are caused by a failure of transmission at the neuromuscular junction (NMJ) [1]. This disease is classified as an autoimmune disease, and antibodies against acetylcholine receptor (ACh-R) and muscle-specific receptor tyrosine kinase (MuSK) [2] at the NMJ play an important role in the pathogenesis. Among patients with MG, the positive rate for ACh-R antibody $(\mathrm{Ab})$ is approximately $80 \%$ [3]. Patients showing ACh-R Abnegative results are considered to show seronegative-type MG (SNMG). Positive results for MuSK Ab are seen in 30-40\% of SNMG cases [2,4]. The remaining cases of MG (5-10\%) show neither ACh-R $\mathrm{Ab}$ nor MuSK Ab. This double-seronegative MG (DSN-MG) includes various types of MG. Among some cases of SNMG (especially ocular $\mathrm{MG}$ ), it is estimated that clustered ACh-R antibodies are not detected by conventional examination because of its low sensitivity [5].

For the treatment of MG, pyridostigmine, predonisolone (PSL), and other immunosuppressants are usually used. However, over the prolonged clinical course of MG, the effects of these drugs often weaken. Under such circumstances, it is difficult to apply and continue optimal therapy for the individual patient with $\mathrm{MG}$, and other therapeutic methods must be sought.

Here, we present a refractory case of late-onset DSN-MG treated with both polypharmacy and plasma exchange (PE), and discuss the therapeutic process in this case.

\section{Case Report}

A 62-year-old man was admitted because of dyspnea, bulbar symptoms (dysphagia and dysarthria), and severe muscular weakness of the limbs starting about a month earlier.

He had felt more easily fatigued at 50 years old. At 52 years old, bilateral blepharoptosis developed. About 1 year later, he consulted Ohmihachiman Municipal Hospital, the predecessor of our hospital, Ohmihachiman Community Medical Center (OCMC), and was diagnosed with MG. After establishing the diagnosis, administration of pyridostigmine was started at $60 \mathrm{mg} /$ day. PSL at $5 \mathrm{mg} /$ day was subsequently added and increased to $25 \mathrm{mg}$ /day in a stepwise manner within a year. About 8 months after diagnosis, thymectomy was performed in another hospital. The pathological diagnosis was normal thymus. Some years later, a complication of steroid-induced diabetes mellitus (DM) was identified. We therefore controlled the dose of PSL in consideration of the degree of symptoms in both MG and DM. At 56 years old, tacrolimus was added, but was stopped 3 years later because of side effects. Ciclosporin (CyA) was then started at $25 \mathrm{mg} /$ day, and was eventually increased to $200 \mathrm{mg}$ /day.

On admission, general findings were almost intact. In terms of abnormal neurological findings, consciousness was alert, and severe bilateral blepharoptosis was noted. Extraocular movements were limited in all directions. He reported diplopia, dysarthria, and dysphagia. Soft palate elevation remained intact, but tongue protrusion was not performed. Muscular weakness was apparent symmetrically in both upper and lower extremities, and the patient could not walk or remain standing. Drugs for MG administered at that time were as follows: pyridostigmine, $240 \mathrm{mg} / \mathrm{day}$; PSL, $15 \mathrm{mg} / \mathrm{day}$; and CyA, 200 $\mathrm{mg} /$ day. Laboratory data showed: white blood cells, $13,100 / \mu \mathrm{L}$; hemoglobin A1c, 7.1\%; and negative results for both ACh-R-Ab and 
MuSK Ab. Thyroid function was normal. The edrophonium $\mathrm{Cl}$ test yielded positive results (Figure 1). On contrast-enhanced computed tomography of the thorax, neither recurrent nor ectopic thymoma was evident. No malignant tumors were detected. Repetitive electrophysiological stimulation of representative muscles showed no findings of waning.

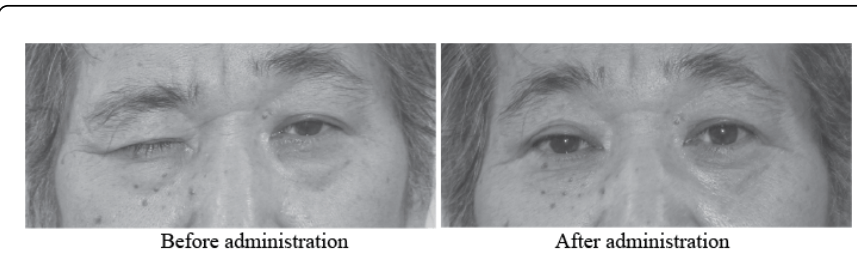

Figure 1: Edrophonium $\mathrm{Cl}$ test (A comparison of before and after administration; improvement of blepharoptosis is obvious).

During the 12 years since onset, thymectomy had been performed and many drugs for MG had already been used. Because of these circumstances, increasing or adding drugs was difficult. Based on this background, as a new method, we decided to perform PE. The effect of double-filtration plasmapheresis (DFPP) on symptoms of MG was initially marked, and this approach was repeated 5 times within this admission.

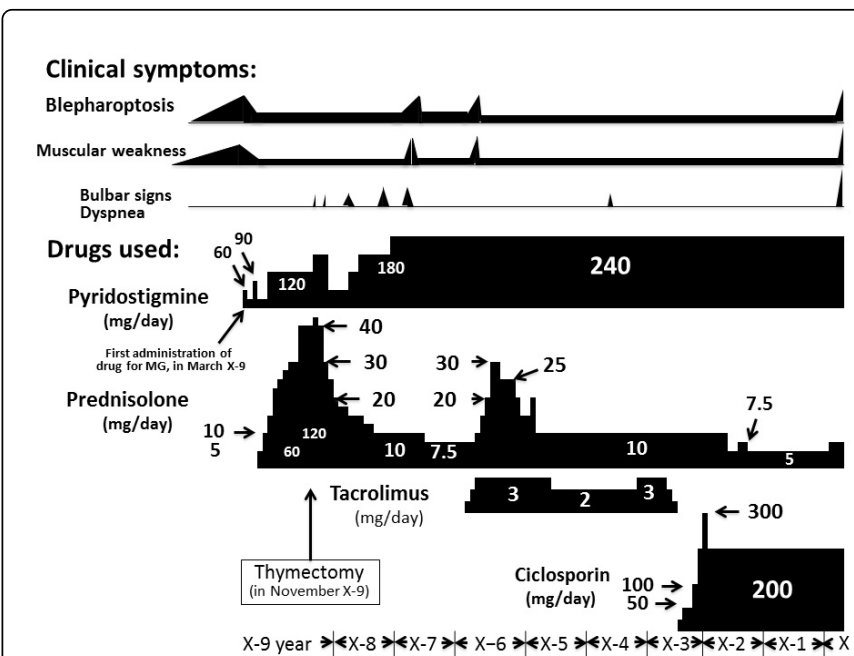

Figure 2: Clinical course and therapeutic process (before DFPP): This figure summarizes the approximately 10 years from onset of MG until the introduction of DFPP. The transitions of main clinical symptoms and administered drugs are shown in detail. About one year after onset, drug administration for MG (pyridostigmine 60 $\mathrm{mg} /$ day) was started in March, 9 years before this admission. Thymectomy was performed in November of the same year.

On discharge, the patient continued with administration of conventional drugs for MG. We investigated the duration of the effect after repeated DFPP. About a month later, symptoms of MG gradually worsened and he became unable to walk. He was then admitted to our hospital again about 2 months after discharge. During this second admission, DFPP was performed a total of 4 times. Although highdose methyl-predonisolone (m-PSL) was additionally administered only once, no clear effect was obtained. In anticipation of frequent
DFPP in the future, surgical construction of an internal shunt for blood dialysis was performed. About 1 month later, the patient was admitted for the third time. During this admission, DFPP was again performed a total of 4 times. Only 3 days after discharge from this third admission, he returned and was admitted to OCMC because symptoms of weakness had again worsened (fourth admission). This time, DFPP was repeated 6 times. After discharge, we decided to perform DFPP once a week. For about 6 months, his clinical condition remained stable. However, during the latter half of this period, the duration of effective response was reducing. On the day before DFPP, muscle weakness was marked.

To help identify a good method for breaking this deadlock, the patient was transferred from OCMC to the Department of Neurology at Kyoto Prefectural University of Medicine (KPUM). The treatment plan was then discussed and reconsidered. The approach put into practice was a stepwise increase in PSL with use of insulin. Specifically, PSL was increased from $15 \mathrm{mg} /$ day to $60 \mathrm{mg} /$ day. The duration of effective response to DFPP subsequently increased, and the frequency of DFPP was able to be decreased. Finally, DFPP was reduced from once a week to once every 2 weeks. After the patient's general condition with this combined therapy stabilized, he returned to OCMC and the daily dose of PSL was gradually tapered.

Shortly afterwards, his condition rapidly deteriorated due to hepatic failure. This deterioration was too fast to initiate therapeutic measures, and methods to improve his condition were unavailable. Despite intensive care, he died about 3 weeks later, about 13 years since the onset of MG, and about 16 months after the introduction of DFPP. The clinical course and therapeutic process before and after introduction of DFPP are summarized in Figures 2 and 3.

\section{Discussion}

This case of MG was characterized by late onset, long clinical course, double-seronegative type, and slow development of drug resistance.

According to a previous report, DSN-MG is characterized by a broader spectrum for age of onset and significantly milder symptoms (compared to MuSK-positive, seronegative MG) [6]. The features of MG in this category are heterogeneous because of the variety in mechanisms of pathogenesis and clinical phenotype. Unknown or unidentified antibodies appear likely to play a pathogenic role in the expression of MG. Some reports have suggested that the clinical features of DSN-MG resemble those of seropositive MG [7,8]. In fact, conventional pharmacotherapy and thymectomy showed some degree of efficacy against symptoms of MG in this case.

Because the deterioration at first admission was regarded as MG crisis, quick and effective measures were required to overcome this medical situation. We decided to perform PE, as a well-known and reasonable therapeutic method for refractory MG $[9,10]$. There are three choices for practical methods of PE. Simple PE is expected to achieve efficacy, but a large volume of very expensive fresh frozen plasma is required. If this method repeated often, the medical costs become enormous. Immunoadsorption plasmapheresis (IAPP) shows therapeutic effect by removing Ach-Ab in cases of seropositive MG [11], but it remains unclear whether IAPP would prove effective against SN-MG, as in the present case. Finally, we adopted DFPP in consideration of the physical and economic burden on the patient. If DFPP had proven ineffective, simple PE would have been adopted as the second choice. Although DFPP was started tentatively, the initial 
effect was marked. Even when the duration of response with DFPP was getting shorter, the effect itself did not decline much. As a result, DFPP was repeatedly performed in this case.

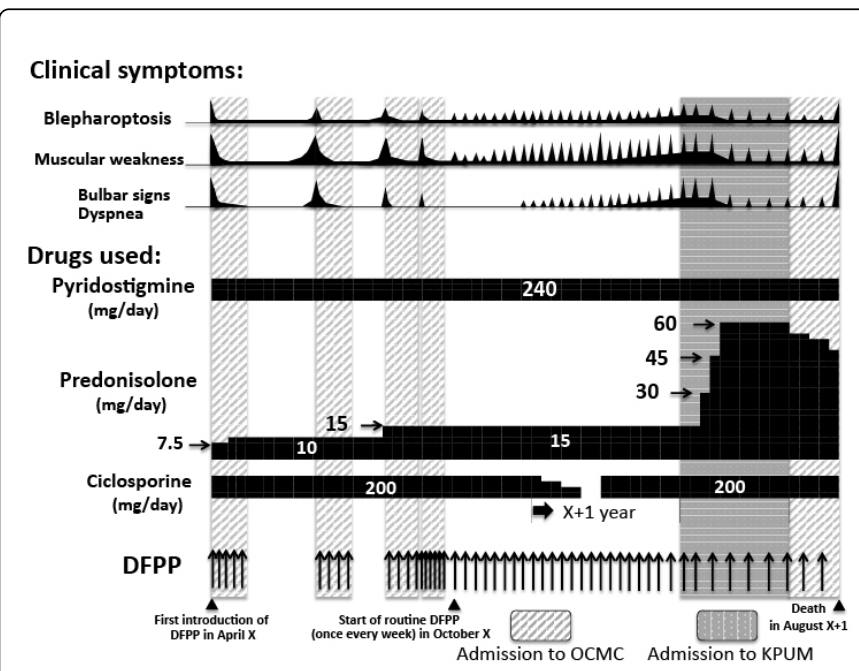

Figure 3: Clinical course and therapeutic process (after DFPP) (DFPP: Double-filtration plasmapheresis; OCMC: Ohmihachiman Community Medical Center; KPUM: Kyoto Prefectural University of Medicine). This figure summarizes the approximately 16 months from first introduction of DFPP until the patient's death. At first, a series of DFPP administrations brought improvements of MG symptoms. However, the duration of the effect was gradually shortened. About 6 months after first introduction of DFPP (in October, X), routine DFPP (once every week, without admission) was started, though it eventually became ineffective. On admission to KPUM, stepwise increases in PSL allowed for prolongation of the interval between DFPP administrations.

Because PE shows effects in the short term rather than the long term [12], few reports have described long-term use of this method for MG. Rodnitzky et al. presented 2 cases of MG in which PE was continued for 5 years. The outcome in each patient was good, and both patients were able to reduce the dose of PSL [13]. Yeh et al. presented a case in which DFPP was continued for 2.5 years. When the duration of response gradually decreased, intravenous immunoglobulin therapy was introduced and the duration of response was prolonged [14]. Lebrun et al. reported 6 cases of refractory MG in which treatment with rituximab proved successful. They described a representative case in which PE had been continued for 6 months [15].

In the present case, we investigated low-density lipoprotein receptor-related protein (Lrp) 4 [16] in serum, showing a negative result. Considering that DFPP each time proved effective and the patient got some improvements, some unknown and unclear antibodies seems likely to be involved in the expression of MG symptoms in such a complicated case. The discovery and identification of antibodies responsible for DSN-MG is a problem to be solved in the future.
In conclusion, DFPP is worth trying as a useful, non-transient treatment in DSN-MG, although the optimal therapeutic method for this type of MG has not been established. Concerning the treatment for such refractory cases of MG, reaching and sustaining synergistic effects with each method as much as possible is important. We should thus consider the optimal combination and utilization depending on the medical situation of the individual patient with MG. We should also pay attention to the balance of effects and side effects from administered drugs and physical burden on the patient.

\section{References}

1. Drachman DB (1994) Myasthenia gravis. N Engl J Med 330: 1797-1810.

2. Hoch W, McConville J, Helms S, Newsom-Davis J, Melms A, et al. (2001) Auto-antibodies to the receptor tyrosine kinase MuSK in patients with myasthenia gravis without acetylcholine receptor antibodies. Nat Med 7: 365-368.

3. Lindstrom JM, Seybold ME, Lennon VA, Whittingham S, Duane DD (1976) Antibody to acetylcholine receptor in myasthenia gravis. Prevalence, clinical correlates, and diagnostic value. Neurology 26: 1054-1059.

4. Shiraishi H, Motomura M, Yoshimura T, Fukudome T, Fukuda $\mathrm{T}$, et al. (2005) Acetylcholine receptors loss and postsynaptic damage in MuSK antibody-positive myasthenia gravis. Ann Neurol 57: 289-293.

5. Jacob S, Viegas S, Leite MI, Webster R, Cossins J, et al. (2012) Presence and pathogenic relevance of antibodies to clustered acetylcholine receptor in ocular and generalized myasthenia gravis. Arch Neurol 69: 994-1001.

6. Evoli A, Tonali PA, Padua L, Monaco ML, Scuderi F, et al. (2003) Clinical correlates with anti-MuSK antibodies in generalized seronegative myasthenia gravis. Brain 126: 2304-2311.

7. Deymeer F, Gungor-Tuncer O, Yilmaz V, Parman Y, Serdaroglu P, et al. (2007) Clinical comparison of anti-MuSK- vs anti-AChR-positive and seronegative myasthenia gravis. Neurology 68: 609-611.

8. Padua L, Tonali P, Aprile I, Caliandro P, Bartoccioni E et al. (2006) Seronegative myasthenia gravis: comparison of neurophysiological picture in MuSK+ and MuSK- patients. Eur J Neurol 13: 273-276.

9. Pinching AJ, Peters DK (1976) Remission of myasthenia gravis following plasma-exchange. Lancet 2: 1373-1376.

10. Kornfeld P, Ambinder EP, Mittag T, Bender AN, Papatestas AE, et al. (1981) Plasmapheresis in refractory generalized myasthenia gravis. Arch Neurol 38: 478-481.

11. Shibuya N, Sato T, Osame M, Takegami T, Doi S, et al. (1994) Immunoadsorption therapy for myasthenia gravis. J Neurol Neurosurg Psychiatry 57: 578-581.

12. Newsom-Davis J, Vincent A (1979) Combined plasma exchange and immunosuppression in myasthenia gravis. Lancet 2: 688 .

13. Rodnitzky RL, Bosch EP (1984) Chronic long-interval plasma exchange in myasthenia gravis. Arch Neurol 41: 715-717.

14. Yeh JH, Chiu HC (1999) Sequential plasmapheresis and intravenous immunoglobulin therapy for refractory myasthenia gravis: case report. Ther Apher 3: 271-274.

15. Lebrun C, Bourg V, Tieulie N, Thomas P (2009) Successful treatment of refractory generalized myasthenia gravis with rituximab. Eur J Neurol 16: 246-250.

16. Higuchi O, Hamuro J, Motomura M, Yamanashi Y (2011) Autoantibodies to low-density lipoprotein receptor-related protein 4 in myasthenia gravis. Ann Neurol 69: 418-422. 